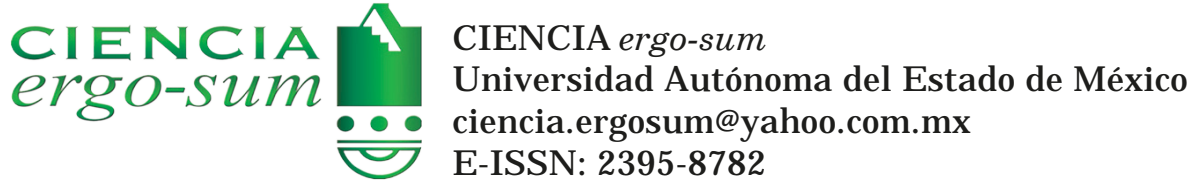

\title{
Conocimiento tradicional y contenido nutricional de Salmea scandens
}

Pérez J osé, Carolina; García Morales, Pedro Antonio; Velasco-Velasco, Vicente Arturo; Hernández Santiago,
Ernesto; Ruiz Luna, J udith; Rodríguez Ortiz, Gerardo
Conocimiento tradicional y conteni do nutricional de Salmea scandens
CIE NCIA ergo-sum, vol. 27, núm. 1, marzo-junio 2020| e76
Universidad Autónoma del Estado de México, México

Pérez J osé, C., García Morales, P. A., Velasco-Velasco, V. A., Hernández Santiago, E., Ruiz Luna, J . y Rodríguez Ortiz, G. (2020). Conocimiento tradicional y contenido nutricional de Salmea scandens. CIE NCI A ergo-sum, 27(1). https://doi.org/10.30878/ces.v27n1a8 


\title{
Conocimiento tradicional y contenido nutricional de Salmea scandens
}

Traditional knowledge and nutritional content of Salmea scandens

\author{
Carolina Pérez José \\ Tecnológico Nacional de México/Instituto Tecnológico del Valle de Oaxaca, México \\ capejo05@gmail.com \\ Pedro Antonio García Morales \\ Tecnológico Nacional de México/Instituto Tecnológico del Valle de Oaxaca, México \\ gamp07@outlook.com \\ Vicente Arturo Velasco-Velasco \\ Tecnológico Nacional de México/Instituto Tecnológico del Valle de Oaxaca, México \\ vicvel5@hotmail.com \\ Ernesto Hernández Santiago \\ Tecnológico Nacional de México/Instituto Tecnológico del Valle de Oaxaca, México \\ jipuri@uotlook.com \\ Judith Ruiz Luna \\ Tecnológico Nacional de México/Instituto Tecnológico del Valle de Oaxaca, México \\ judithruizl@hotmail.com \\ Gerardo Rodriguez Ortiz \\ Tecnológico Nacional de México/Instituto Tecnológico del Valle de Oaxaca, México \\ geraro65@gmail.com
}

\section{RESUMEN}

Salmea scandens, conocida como palo de chile, es una planta económicamente importante en la región costa de Oaxaca. Con el objetivo de documentar el conocimiento tradicional de su uso y el contenido de nutrientes de la parte comestible, se realizó un estudio en Buena Vista Loxicha, Pochutla, Oaxaca, por medio de entrevistas semiestructuradas. Se recolectaron muestras de tallo para cuatificar el contenido de fibra cruda, grasa, proteína y minerales. Se encontraron dos morfotipos: palo de chile blanco y palo de chile negro, con usos comestible y medicinal. De las plantas se consume el cámbium vascular del tallo, preferentemente hervido, que contiene $9.44 \%$ de fibra cruda, $2.51 \%$ de grasa y $3.10 \%$ de proteína. El mineral más abundante fue el potasio con $505.53 \mathrm{mg} 100 \mathrm{~g}^{-1}$.

Palabras CLAVE: morfotipos, nutrientes, palo de chile.

\begin{abstract}
Salmea scandens, known as chili stick, is an economically important plant in the Oaxaca coast region. To document the traditional knowledge of its use and the nutritional content of the edible part, the present study was conducted in Buena Vista Loxicha, Pochutla, Oaxaca, Mexico. Semi-structured interviews were applied. In stem, crude fiber, fat, protein and minerals were quantified. Two morphotypes, chili white stick and chili black stick were found, with edible and medicinal uses. Vascular stem cambium is eaten, preferably boiled, which contains $9.44 \%$ of raw fiber, $2.51 \%$ fat and $3.10 \%$ protein es consumed. The most abundant mineral was potassium with $505.53 \mathrm{mg} 100 \mathrm{~g}-1$.
\end{abstract}

KEYWORDS: morphotypes, nutrients, chili stick. 


\section{INTRODUCCIÓN}

El estado de Oaxaca alberga la mayor biodiversidad florística de México (Bastida-Zavala et al., 2013). Se calcula que en el país existen alrededor de 30000 especies de plantas vasculares, lo que hace del país una de las zonas florísticamente más ricas del planeta (Casas et al., 1994). Desde sus orígenes, el desarrollo de las sociedades humanas y su cultura tienen relación con el conocimiento de la biodiversidad y del uso de una gran cantidad de especies vegetales (Rendón et al., 2001), que incluye plantas medicinales y comestibles (Bautista-Cruz et al., 2011).

Las comunidades rurales de México comúnmente complementan su alimentación con plantas comestibles recolectadas en la vegetación que circunda su territorio. Al respecto, estudios etnobotánicos en distintas regiones del país han documentado más de 1500 especies de plantas comestibles silvestres que llegan a constituir entre 8 y 17\% de la dieta anual de las familias campesinas (Lascurain et al., 2010), y además contienen elementos nutritivos de alto valor, como vitaminas, sales minerales y otras sustancias bioactivas que tienen efectos sobre el cuerpo humano (Bourges y Vargas, 2015).

Las plantas comestibles silvestres al crecer en estado natural conservan todas sus propiedades benéficas (Castillo y Cáceres, 2009). Los bejucos son de gran importancia para obtener medicinas, estimulantes, fibras y alimentos (Burelo et al., 2009). Salmea scandens DC es una planta silvestre de hábito arbustivo trepador (bejuco), conocido como palo de chile (Luna-José y Rendón-Aguilar, 2008; Juárez-Catañeda, 2013; INEE, 2014) de gran importancia en la Sierra Sur y costa del estado de Oaxaca.

Buena Vista Loxicha, Pochutla, es una localidad oaxaqueña con riqueza cultural en donde los pobladores usan plantas y animales para satisfacer necesidades comestibles y medicinales. En esta localidad, $S$. scandens adquiere importancia por ser una planta comestible silvestre y porque posee un efecto similar al de la anestesia propiciando que se utilice como un sustituto del chile debido al efecto que ejerce. Es importante que este patrimonio biocultural sea documentado, inventariado, comprendido y vigorizado por la ciencia en la búsqueda de modelos alternativos de manejo de recursos naturales, salud, vivienda, cultura, visiones y concepciones del mundo (Conacyt, 2012).

Por lo anterior, el propósito de este artículo es conocer el contenido nutricional de $S$. scandens y documentar el conocimiento tradicional para obtener información referente a la cantidad de nutrientes que aporta en la dieta de los pobladores.

\section{Materiales y métodos}

\section{1. Área de estudio}

El estudio se realizó en la localidad de Buena Vista, municipio de San Agustín Loxicha, Distrito de San Pedro Pochutla, región Sierra Madre del Sur en la planicie costera del Pacífico Sur de Oaxaca. Se localiza en las coordenadas $16^{\circ} 01^{\prime}$ latitud norte y $96^{\circ} 37^{\prime}$ longitud oeste, a una altitud de $1820 \mathrm{~m}$ (Plan Municipal de Desarrollo Rural Sustentable de San Agustin Loxicha, Pochutla, Oaxaca, 2008-2010). El consumo de S. scandens en la localidad data de muchos años atrás, incluso personas de edad avanzada lo reportan como costumbre de sus padres y abuelos.

\subsection{Aplicación de entrevistas y muestreo de ejemplares}

Con el método etnográfico, que consiste en aplicar entrevistas semiestructuradas y de observación participante (Murillo y Martínez, 2010), participaron 118 personas que representan 12.7\% del total de los habitantes de Buena Vista Loxicha. Los intervalos de edad considerados fueron de 15-20, 21-30, 31-40, 41-50, 51-60, 61 y más años y así identificar sus conocimientos sobre la especie en estudio de acuerdo con su edad. El trabajo se llevó a cabo de febrero de 2015 a mayo de 2016. 
Se recolectaron cinco plantas en cada una de las tres altitudes: a 506 m (estrato bajo), entre 1208 y $1310 \mathrm{~m}$ (estrato medio) y entre 2124 y $2135 \mathrm{~m}$ (estrato alto). Estas plantas se describieron con la clave dicotómica de la Flora del Bajío, fascículo 157 (Rzedowski et al., 2011). También se obtuvieron datos morfológicos como forma de crecimiento, tipo de raíz, desprendimiento del cambium vascular, nerviación, textura de la hoja, filotaxia, tipo de inflorescencia, número de flores por inflorescencia; asimismo, con un vernier se midió el ancho de lámina foliar $(\mathrm{cm})$, la longitud y ancho de corola $(\mathrm{cm})$, la longitud y ancho de cáliz $(\mathrm{cm})$ y la longitud de palea $(\mathrm{cm})$. Para comparación con $S$. scandens, se recolectó la planta Salmea oligocephala Hemsl por ser una especie con la misma clasificación taxonómica y presentar características singulares a la especie de estudio. La descripción morfológica se realizó considerando el promedio de las plantas recolectadas.

\section{3. Manejo y análisis de datos}

Se realizó un análisis de conglomerados para la agrupación de los morfotipos mediante el método de distancia promedio y análisis de componentes principales. Se utilizó el programa NCSS (Number Cruncher Statistical System) demo en internet 2017.

Los ejemplares se agruparon de la siguiente manera: SM1, ejemplares de estrato bajo; SM2, ejemplares de estrato medio; SM3, ejemplares de estrato alto y SO, S. oligocephala planta testigo.

\section{4. Cuantificación de nutrientes}

El cámbium vascular (parte comestible) de diez plantas de S. scandens tomadas al azar se analizó de forma cruda y cocida. Cruda se refiere a que el cámbium vascular se consume directamente al desprenderlo del tallo; para la cocida se llevó un proceso de hervor en agua durante $30 \mathrm{~min}$. Posteriormente se secaron las muestras a $60{ }^{\circ} \mathrm{C}$ durante 48 h en una estufa con circulación de aire forzado (Riossa $\left.{ }^{\circledR}\right)$ y después se molieron.

Para las determinaciones en el laboratorio se aplicaron las técnicas de la Asociación Oficial de Química Analítica (AOAC, 1984). La fibra cruda se determinó con la técnica de digestión ácido-base; la grasa cruda se obtuvo con el método Soxhlet; la concentración de nitrógeno total, por el método microKjeldahl; el fósforo mediante el método del vanadato-molibdato amarillo; en un espectrofotómetro UV-VIS (Cintra 10®); los macroelementos, $\mathrm{Na}, \mathrm{K}, \mathrm{Ca}, \mathrm{Mg}$ y microelementos, $\mathrm{Cu}, \mathrm{Fe}, \mathrm{Mn}$ y Zn, por espectrofotometría de absorción atómica (EAA) (Thermo Scientific ICE 3000 series $\left.{ }^{\circledR}\right)$. Estas determinaciones se llevaron a cabo en el Laboratorio de Diagnóstico Ambiental del Instituto Tecnológico del Valle de Oaxaca (ITVO).

\section{Resultados Y Discusión}

\section{1. Conocimiento tradicional}

De las personas entrevistadas, 69 fueron mujeres y 49 hombres de 15 años en adelante, debido a que es poco común que los niños consuman dicha especie. Se obtuvieron dos usos de S. scandens: comestible (63\%) y medicinal (37\%), los cuales representan la relación que tienen los pobladores de la localidad con la especie en estudio, así como el conocimiento cultural que poseen sobre la misma.

Para recolectar los tallos de $S$. scandens, las personas caminan aproximadamente de dos a cuatro horas. Este tallo puede encontrase todo el año en las partes altas; sin embargo, en los meses de junio a septiembre presentan un mayor volumen o son más suculentos y la corteza se levanta con facilidad, lo cual ayuda a identificar que es momento de recolectarla; por este motivo la venta es mayor en esta temporada.

Se vende por pieza de aproximadamente $50-80 \mathrm{~cm}$ de largo con valor entre 2 y 5 pesos dependiendo del grosor que tenga (diámetro de 1.5 a $3 \mathrm{~cm}$ ) y su valor aumenta debido a que presenta mayor cantidad de cámbium 
vascular. De los consumidores, $54 \%$ lo compra en mercados locales o con personas que venden a domicilio, 35\% lo recolecta y sólo $11 \%$ lo siembra.

Las personas lo consumen por tradición, costumbre y por sabor, ya que es un sustituto del chile. Provoca adormecimiento en la lengua y cierta irritación en la boca. Por lo anterior, las personas le han denominado "palo de chile", que en dialecto zapoteco es "Lus ya'a yin" y también lo conocen como "duerme boca” o "Ya'a naa 'yoou”. Algunos investigadores (Sosa-Montes et al., 2007; Luna-José y Rendón-Aguilar, 2008) han documentado previamente el consumo del tallo de $S$. scandens en la Sierra Sur de Oaxaca.

En este artículo se encontró que la parte comestible de $S$. scandens es el cámbium vascular del tallo, también conocido como "carne, tela o yaco" (figura 1). Para obtener el cámbium se tiene que retirar la peridermis (figura 2). Las personas lo consumen hervido (82\%), crudo (16\%) y asado (2\%); la forma hervida es la más fácil para desprender el cámbium. La especie es utilizada como sustituto del picante y agente saborizante como lo indican Villa-Ruano et al. (2015), además de consumirse en comidas típicas con frijoles (Phaseolus vulgaris L.), chepiles (Crotalaria longirostrata Hook \& Arn) o como acompañamiento de cualquier otro alimento.

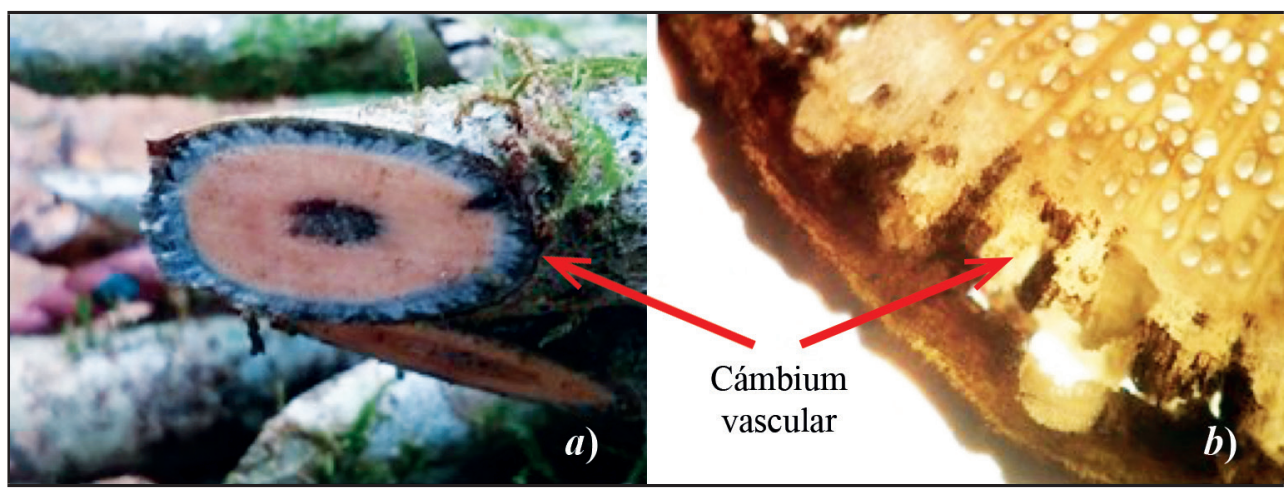

FIGURA 1

Cámbium vascular de $S$. scandens

Nota: $a)$ macroscópica y $b$ ) microscópica

Fuente: fotografía de Carolina Pérez-José.

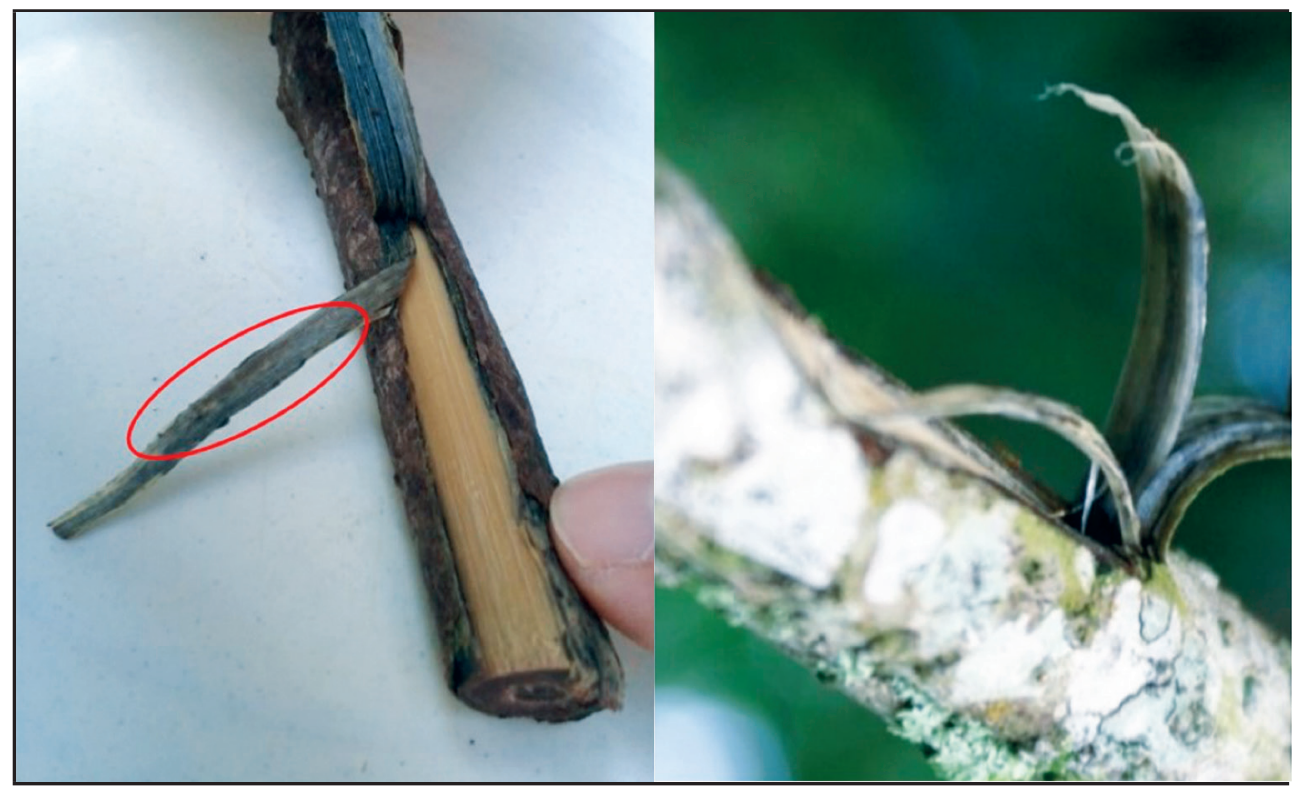

FIGURA 2

Cámbium vascular (parte consumida) de $S$. scandens

Fuente: fotografía de Vicente Arturo Velasco-Velasco. 
Algunas personas mencionaron que al cortar la planta se debe tener cuidado, pues el olor que desprende es fuerte y ocasiona molestias en la piel y en los ojos. Indican también que no hay reportes hasta el momento de personas que hayan sufrido daños severos por esta causa.

De los padecimientos en seres humanos tratados con S. scandens se detectaron 15, clasificados en 9 categorías (cuadro 1), de las cuales las crónico-degenerativas mostraron la mayor frecuencia (34.4\%); dentro de esta categoría, la diabetes fue la más reportada (29.7\%). La planta puede tener diferentes usos: con los tallos se elabora una infusión y se toma como "agua de uso diario" para controlar la diabetes; el cámbium vascular se coloca en la muela que adolece para disminuir el dolor; para curar la mordedura de serpientes, de perro y piquete de alacrán se muelen las hojas y se aplica la mezcla directamente en la herida; para la disminución de la hinchazón en los pies, éstos se sumergen en un recipiente con infusión de hojas y tallos. De Feo (2003) afirma que esta planta es efectiva en el tratamiento de la esterilidad femenina si se bebe de dos a tres tazas al día de una infusión de hoja fresca (aproximadamente $50 \mathrm{~g}$ en 11 de agua); sin embargo, este uso no fue reportado por las personas entrevistadas de la localidad.

\section{CUADRO 1}

Categoría y padecimientos tratados con $S$. scandens por pobladores de Buena Vista Loxicha, Pochutla

\begin{tabular}{|llccc|}
\hline Categoría & Padecimiento & $\begin{array}{c}\text { Frecuencia } \\
\text { Absoluta }\end{array}$ & $\begin{array}{c}\text { Frecuencia relativa } \\
\mathbf{( \% )}\end{array}$ & $\begin{array}{c}\text { Frecuencia por } \\
\text { categoría (\%) }\end{array}$ \\
\hline Crónicas-degenerativas & Diabetes & 19 & 29.7 & 34.4 \\
& Cáncer & 2 & 3.1 & 17.2 \\
Lesiones & Presión & 1 & 1.6 & 14.0 \\
Odontológicos & Dolor de muela & 11 & 17.2 & 14.0 \\
Inflamación & Hinchazón & 9 & 14.0 & 9.2 \\
Síndrome de & Aire & 9 & 14.0 & \\
filiación cultural & Latido & 3 & 4.6 & 4.8 \\
\hline & Acidez & 3 & 4.6 & \\
Digestivos & Anginas & 1 & 1.6 & 3.2 \\
& Granos en la boca & 1 & 1.6 & 1.6 \\
Dolor & Pie & 1 & 1.6 & 1.6 \\
Infecciones & Cabeza & 1 & 1.6 & 100 \\
\hline Musculares & Tos & 1 & 1.6 & 1.6 \\
\hline Total & Hernias & 1 & 1.6 & 100 \\
\hline
\end{tabular}

Fuente: elaboración propia.

La información proporcionada por los habitantes de la localidad y sus observaciones son empíricas, puesto que faltan estudios donde se demuestren las propiedades medicinales de $S$. scandens. En San Luis Potosí es llamada "chilmecate o chilmecatl en náhuatl” y significa "hierba de la muela" (Martínez, 1994; Rzedowski et al., 2011), también se conoce como "duerme boca” en Colombia y “bejuco de muela” en Puerto Rico (Herz y Kulanthaivel, 1985) precisamente porque disminuye este malestar, padecimiento que tratan los pobladores de Buena Vista, Loxicha con el palo de chile. 
En América Central se le atribuye un uso medicinal y está documentado como una planta de la etnomedicina oaxaqueña (Villa-Ruano et al., 2013; Villa-Ruano et al., 2015). Herz y Kulanthaivel (1985) mencionan que en la medicina indígena tiene un uso aparentemente como un anestésico por el efecto entumecedor en la lengua y por la presencia de algunos compuestos como el sitosterol, estigmasterol, ácido linolénico y esteárico, una pequeña cantidad de costunólido y dos compuestos isómeros inestables de fórmula $\mathrm{C}_{16} \mathrm{H}_{25} \mathrm{NO}$

Los pobladores clasifican a $S$. scandens por tres factores: $a$ ) por el color del cámbium vascular se conocen como palo de chile blanco y palo de chile negro; $b$ ) por la ubicación geográfica, del "cerro", son plantas que se encuentran a más de $1000 \mathrm{~m}$ de altitud y de la "costa" se encuentran a menos de $550 \mathrm{~m}$ de altitud y c) por el color de las flores en blancas y moradas.

La planta muestra el crecimiento en forma de bejuco, las raíces son adventicias y los tallos herbáceos y leñosos son redondos con lenticelas vistosas. El cámbium vascular muestra consistencia carnosa; las hojas muestran peciolo acanalado $(1.56-2.42 \mathrm{~cm})$, lámina ovada-lanceolada $(11.33 \times 4.58 \mathrm{~cm}, 12.67 \times 6.58 \mathrm{~cm})$, el ápice es acuminado y agudo-obtuso, la base es redondeada o decurrente, el margen es especialmente dentado a casi entero, la textura es coriácea y glabra; la filotaxia es decusada. Las inflorescencias muestran forma de panícula cimosa, tienen de 7.7 a $8 \mathrm{~mm}$ de largo $\times 4.3$ a $6.9 \mathrm{~mm}$ de ancho, flores blancas campanuladas-gamopétalas de 21 a 37, en algunas plantas la prefloración presenta un color morado; corola de 2.9 a $3 \mathrm{~mm}$ de largo por 1.5 a $1.8 \mathrm{~mm}$ de ancho; vilano de 3 a $3.2 \mathrm{~mm}$ de largo $\times 1.4$ a $1.5 \mathrm{~mm}$ de ancho. Las plantas encontradas de $1208 \mathrm{~m}$ a $2135 \mathrm{~m}$ de altitud mostraron cáliz de color morado en la etapa de prefloración, y en el caso de las plantas encontradas a una altitud de $506 \mathrm{~m}$ no se presentaba esta característica (figura 3). Las características morfológicas son similares con estudios de Bolick (1991) y Rzedowski et al. (2011), a excepción del color de la floración.

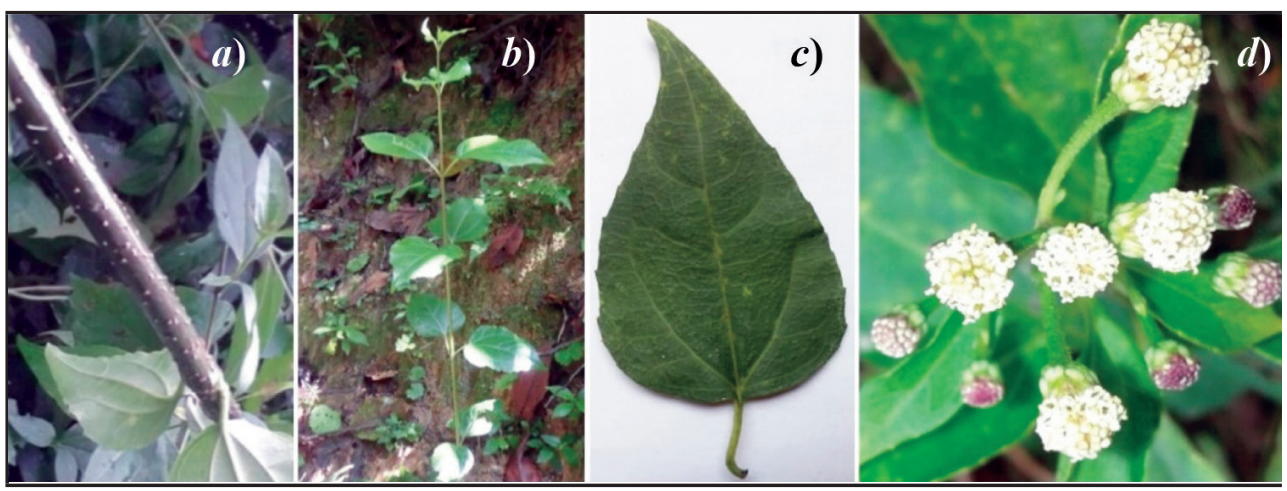

FIGURA 3

a) Lenticelas, $b$ ) filotaxia, $c$ ) hoja y $d$ ) inflorescencia de $S$. scandens Fuente: fotografía de Pedro Antonio García Morales.

Salmea oligocephala (SO) se separa estadísticamente de los morfotipos de $S$. scandens con distancia media de 40.37 (gráfica 1), esto es, porque se tratan de especies diferentes. SM2 y SM3 presentan distancia media de 1.73 respecto de SM1 con distancia media de 7.46. A pesar de tratarse de la misma especie, SM2 y SM3 tienen características similares entre sí, ya que son de la variedad palo de chile blanco y presentan diferencias con SM1 que representa a la variedad palo de chile negro. Morales (2007) en un estudio realizado en Santa María Xadani, San Miguel del Puerto, Oaxaca, reportó la presencia de dos variedades de palo de chile.

Las características morfológicas que influyeron en la diferenciación de esta especie de acuerdo con el análisis de componentes principales, se reflejaron en la forma de crecimiento, el tallo, ancho de lámina, nervadura de la hoja y textura de la hoja, donde la forma de crecimiento (75.23) es la de mayor relevancia. 


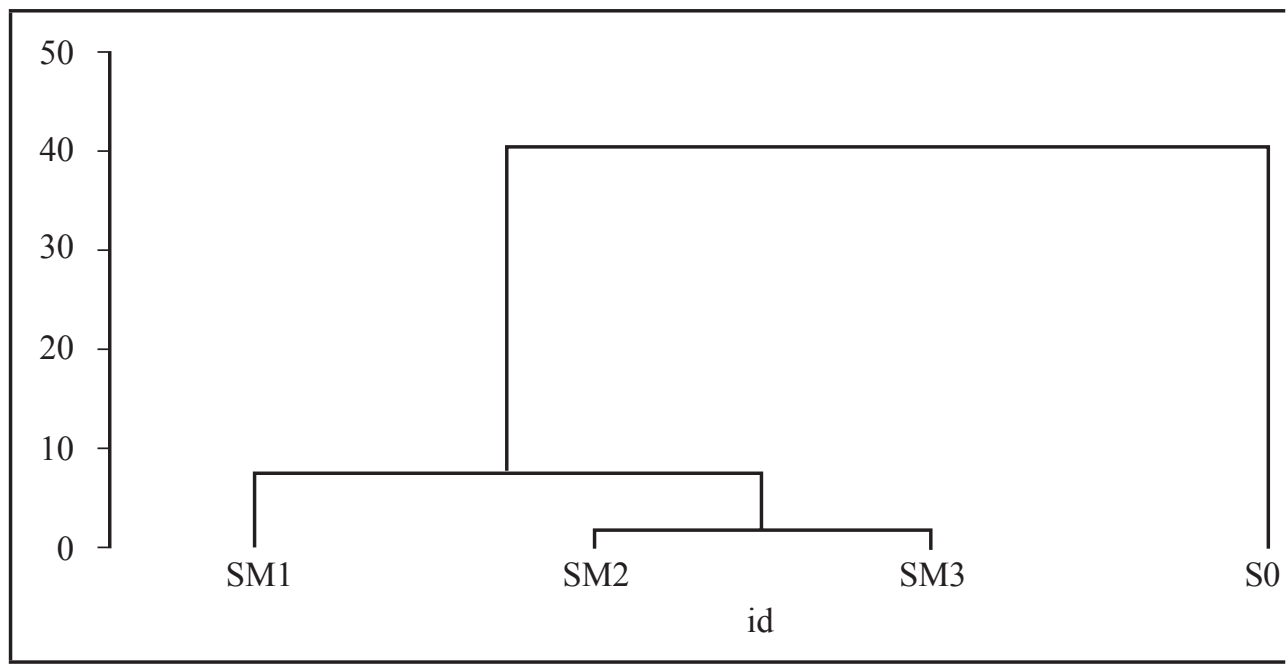

GRÁFICA 1

Dendrograma de la agrupación de S. oligocephala y las variedades de $S$. scandens Fuente: elaboración propia.

\section{Contenido nUtricional}

Para obtener la parte comestible -el cámbium vascular del tallo en $S$. scandens- las personas de Buena Vista, Loxicha, hierven los tallos o lo extraen de forma cruda, pues son las principales formas de consumo.

Los análisis del cámbium vascular mostraron que el contenido de fibra cruda, grasa y proteína fueron mayores en muestras crudas respecto a las muestras hervidas en 16.2, 17.1 y $58.7 \%$ respectivamente; asimismo, registraron valores mayores en $\mathrm{P}, \mathrm{K}, \mathrm{Mg}$, $\mathrm{Mn}$ y $\mathrm{Zn}$ y valores menores en $\mathrm{Ca}, \mathrm{Cu}$, Fe y $\mathrm{Na}$ respecto de las muestras cocidas (cuadro 2). Lo anterior puede deberse a que durante la cocción se incrementa la temperatura ocasionando reacciones físicas y químicas (Castro-González et al., 2013), lo cual es común en verduras y hortalizas (Dapcich et al., 2004), así como el arrastre de los nutrientes desde el alimento hacia el medio de cocción, fenómeno conocido como lixiviación (Basulto et al., 2014).

Además, los alimentos varían en su contenido de nutrientes, según la variedad particular del alimento y las condiciones en las que se produce, almacena y prepara (FAO, 2013a). El aporte nutrimental de un producto depende de su contenido de nutrimentos y de la cantidad que se consume de dicho alimento (Bourges, 2011).

Observando el contenido nutricional en otros cultivos, $S$. scandens es menor en fibra cruda respecto a $P$. vulgaris, mayor en grasa respecto a $P$. vulgaris, $Z$. mays y $S$. lycopersicum; en proteína es mayor a $S$. lycopersicum y menor a P. vulgaris y $Z$. mays (cuadro 2). Escudero y González (2006) indican consumir de 20 a 35 g/ día de fibra de diferentes fuentes. Gonzáles-Torres et al. (2007) recomiendan de 40 a 60 g de proteínas al día para un adulto sano. Por otra parte, Hernández (2004) recomienda una ingesta diaria de grasa de 20 a $35 \mathrm{~g}$ y de proteína de 10 a $35 \mathrm{~g}$. Bourges (2011) menciona que el aporte nutrimental de un producto depende del contenido de nutrimentos y de la cantidad que se consume de dicho alimento. En este caso, los alimentos del cuadro 2 se encuentran por debajo de lo recomendado por separado; sin embargo, se complementan en la dieta diaria cuando son acompañados en los platillos.

S. scandens puede considerarse un alimento de bajo contenido calórico, ya que los vegetales son alimentos bajos en lípidos (Lara-Cortés et al., 2014). El contenido de fibra cruda y proteína pueden considerarla como un alimento alternativo con propiedades nutricionales aceptables; el contenido bajo en grasa es una propiedad nutritiva que describe el nivel de un determinado nutriente contenido en un alimento (FAO, 2013b). 
Referente al contenido mineral en otros cultivos, se encontró que la cantidad de $\mathrm{P}$ y Ca es mayor en $Z$. mays respecto a $S$. scandens y $S$. lycopersicum; en contenido de Na, Fe y K, $S$. scandens es mayor que $P$. vulgaris y $S$. lycopersicum y en contenido de $\mathrm{Zn}, S$. scandens es menor que $P$. vulgaris y es mayor respecto a $S$. lycopersicum. Latham (2002) señala que los elementos trazas son necesarios, pero en cantidades mucho menores (menos de $100 \mathrm{mg}$ /día), dentro de los cuales se encuentran el Zn, Cu y Mn. García (2006) menciona que la ingesta diaria recomendada de Fe y $\mathrm{Zn}$ es de 14 y 15 mg respectivamente. La FAO (2006) recomienda la ingesta diaria de $\mathrm{Fe}$, en mujeres de 11-29 mg y en hombres $14 \mathrm{mg}$. Como se observa en el cuadro 2, los alimentos mencionados presentan las cantidades de ingesta recomendadas; en el caso particular del Fe, 100 gramos de $S$. scandens cruda o cocida proporcionan por sí mismas el requerimiento diario de este mineral con una concentración superior a la del frijol, la tortilla y el tomate. Asimismo, respecto al Ca y K, Latham (2002) propone ingerir más de 100 $\mathrm{mg} /$ día, cantidad que $S$. scandens aporta cuando se consume acompañada de otros alimentos. La mayoría de las veces complementa el requerimiento de ingesta diario para estos minerales.

\section{CUADRO 2}

Comparación del contenido nutrimental de Salmea scandens en porción de $100 \mathrm{~g}$ en base fresca en sus principales formas de consumo, respecto al frijol, maíz y tomate

\begin{tabular}{lccccc} 
& \multicolumn{2}{c}{ "Salmea scandens } & *Phaseolus vulgaris & **Zea mays & **Solanum lycopersicum \\
& Cruda & Hervida & Cocido & Tortilla & Crudo \\
\hline Fibra (g) & 10.97 & 9.44 & 12.00 & 4.47 & 1.20 \\
Grasa (g) & 2.94 & 2.51 & 2.50 & 1.00 & 0.30 \\
Proteína (g) & 4.92 & 3.10 & 23.50 & 5.40 & 0.80 \\
$\mathrm{SO}_{4}=(\mathrm{mg})$ & 121.39 & 76.66 & -- & -- & -- \\
$\mathrm{P}(\mathrm{mg})$ & 32.54 & 15.80 & -- & 123.00 & 24.00 \\
$\mathrm{Cu}(\mathrm{mg})$ & -- & 0.07 & -- & -- & -- \\
$\mathrm{Fe}(\mathrm{mg})$ & 13.01 & 22.65 & 6.00 & 0.20 & 0.60 \\
$\mathrm{Mn}(\mathrm{mg})$ & 2.40 & 2.33 & -- & -- & - \\
$\mathrm{Na}(\mathrm{mg})$ & 8.73 & 16.50 & -- & -- & 5.00 \\
$\mathrm{~K}(\mathrm{mg})$ & 834.61 & 505.53 & -- & -- & 237.00 \\
$\mathrm{Ca}(\mathrm{mg})$ & 95.09 & 124.98 & -- & 124.00 & - \\
$\mathrm{Mg}(\mathrm{mg})$ & 39.00 & 34.21 & 200.00 & -- & - \\
$\mathrm{Zn}(\mathrm{mg})$ & 1.16 & 0.76 & 3.30 & -- & 0.17 \\
\hline
\end{tabular}

$\mathrm{SO}_{4}=$ Sulfatos, $\mathrm{P}=$ Fósforo, $\mathrm{Cu}=$ Cobre, $\mathrm{Fe}=$ Hierro, $\mathrm{Mn}=$ Manganeso, $\mathrm{Na}=$ Sodio, $\mathrm{K}=$ Potasio, $\mathrm{Ca}=$ Calcio, $\mathrm{Mg}=$ Magnesio, $\mathrm{Zn}=$ Zinc. Unidad de medida g (gramo) y mg (miligramo). Fuente: "elaboración propia, ${ }^{*}$ Figueroa et al. (2010) y del **INCAP (2007).

\section{AnÁLISIS PROSPECTIVO}

El resultado de la investigación indica que $S$. scandens puede ser una especie de suma importancia, ya que potencialmente puede ser utilizada como alimento alternativo. La información del uso medicinal que proporcionaron los pobladores de la localidad es reveladora en cuanto a las enfermedades mencionadas y tratadas con esta especie para que en futuras investigaciones se indague cuáles son los principios activos que $S$. scandens posee y que ayudarán al tratamiento de diversos padecimientos, por lo que su posible uso en la industria farmacéutica pudiera ser de gran relevancia. Por otra parte, el conocimiento en lo que se 
refiere al contenido de nutrientes, en las dos principales formas en la que la población la consume, permite contar con datos base sobre su importancia como alimento para la concientización sobre el uso racional y aprovechamiento sostenible de esta especie.

\section{Conclusiones}

S. scandens presentó dos usos: comestible y medicinal. El cambium vascular del tallo es la parte que se consume de forma cruda y hervida. Asimismo, se utilizan las hojas y tallos para aliviar enfermedades, principalmente para el control de la diabetes. Existen dos variedades de $S$. scandens conocidas comúnmente como palo de chile blanco y palo de chile negro, la principal característica que las distingue es su sabor y consistencia; la variedad blanca es la que se consume con mayor frecuencia. De acuerdo con el contenido nutricional, se determinó que el cámbium vascular de los tallos en muestras crudas de Salmea scandens posee $1.53 \%$ de fibra cruda, $0.43 \%$ de grasa y $1.82 \%$ de proteína mayor que en las muestras hervidas. En cuanto al contenido de minerales, la concentración mayor se encontró en las muestras crudas, el más abundante fue el potasio $(\mathrm{K})$. Por lo tanto, el consumo en crudo aporta un contenido nutricional mayor que de forma hervida.

\section{ReFERENCIAS}

AOAC (Association Official of Analytical Chemists). (1984). Official methods of analysis. Washington, D.C.

Bastida-Zavala, J. R., García-Madrigal, Ma. del S., Roas-Alquicira, E. F., López-Pérez, R. A., Benítez-Villalobos, F., Meraz-Hernando, J. F., Torres-Huerta, A. M., Montoya-Márquez, A., \& Barrientos-Luján, N. A. (2013). Marine and coastal biodiversity of Oaxaca, Mexico. Check List Journal of Species List and Distribution, 9(2), 329-390.

Basulto, J., Moñino, M., Farran, A., Baladia, E., Manera, M., Cervera, P., Romero-de-Ávila, Ma. D., Miret, F., Astiasarán, I., Bonany, J., Gelabert,V., Ballesteros, J. M., Martínez, A., Palou, A., Labrador, J., Marques-Lopes, I., Russolillo, G., Alonso, M., Ruiquelme, F., Polanco, I., Rodríguez-Artalejo, F. y Martínez, N. (2014). Recomendaciones de manipulación doméstica de frutas y hortalizas para preservar su valor nutritivo. Revista Española de Nutrición Humana y Dietética, 18(2), 100-115.

Bautista-Cruz, A., Arnaud-Viñas, M. R., Martínez-Gutiérrez, G. A., Sánchez-Medina, P. S. \& Pérez-Pacheco, R. (2011). The traditional medicinal and food uses of four plants in Oaxaca, Mexico. Journal of Medicinal Plants Research, 5(15), 3404-3411.

Bolick, M. R. (1991). Systematics of Salmea DC. (Compositae: Heliantheae). Systematic Botany. American Society of Plant Taxonomists, 16(3), 462-477.

Bourges, R. H. (2011). El chile en la alimentación humana. In K. Richterich (Coord.), El chile: protagonista de la Independencia y la Revolución. México: Fundación Herdez A. C.

Bourges, R. H. y Vargas, G. L. A. (2015). La cocina tradicional y la salud. Revista Digital Universitaria, 16(5), 1-11.

Burelo, R. C. M., Guadarrama, O. Ma. de los A., De la Cruz, L. A. M. y Verástegui, H. E. (2009). Generalidades e importancia de las plantas trepadoras y avances en su estudio en el estado de Tabasco. Kuxulkab, 15(28), 5-9.

Casas A., Viveros, J. L. y Caballero, J. (1994). Etnobotánica mixteca. Sociedad, cultura y recursos naturales en la montaña de Guerrero. Consejo Nacional para la cultura y las Artes/Instituto Nacional Indigenista. México.

Castillo, B. M. T. y Cáceres, N. M. T. (2009). El bosque como fuente de alimento: un estudio etnobotánico de plantas silvestres comestibles en tres comunidades de la Reserva Biológica Indio-Maiz, y tres comunidades de la reserva de la Biosfera BOSAWAS. Managua: Universidad Nacional Agraria. 
Castro-González, Ma. I., Maafs-Rodríguez, A. G. y Pérez-Gil, R. F. (2013). Variación del contenido de lípidos y perfil de ácidos grasos en atún, trucha marina y pámpano sometidos a seis técnicas de cocción. Archivos Latinoamericanos de Nutrición, 63 (1),74-86.

Conacyt (Consejo Nacional de Ciencia y Tecnología). (2012). Red de etnoecología y patrimonio biocultural. México, D.F.

Dapcich, V., Salvador, C. G., Ribas, B. L., Pérez, R. C., Aranceta, B. J. y Serra, M. L. (2004). Guía de la alimentación saludable. Madrid: Sociedad Española de Nutrición Comunitaria.

De Feo, V. (2003). Ethnomedical field study in northern Peruvian Andes with particular reference to divination practices. Journal of Ethnopharmacology, 85, 243-256.

Escudero, A. E. y González, S. P. (2006). La fibra dietética. Nutrición Hospitalaria, 21, 61-72.

FAO (Food and Agriculture Organization). (2006). Guia de nutrición de la familia. Roma. p. 137

FAO (Food and Agriculture Organization). (2013a). Anexo 3: Contenido de nutrientes en alimentos seleccionados. Consultado el 18 de junio de 2015. Disponible en http://www.fao.org/docrep /006 /w0073s/w0073s1x.htm.

FAO (Food and Agriculture Organization). (2013b). Directrices para el uso de declaraciones nutricionales. Consultado el 20 de junio de 2015. Disponible en http://www.fao.org/docrep/w8612s/W8612s06.htm.

Figueroa, G. J. J., Guzmán, M. S. H., Herrera, H. M. G., Rumayor, R. A. F., Alvarado, N. M. D. y Sánchez, T. M. G. (2010). Botana a base de frijol con alto valor nutricional y nutracéutico. Folleto Técnico 28. Campo Experimental Zacatecas. CIRNOC-INIFAP. p. 27

García, G., A. (2006). Ingesta de nutrientes: conceptos y recomendaciones internacionales (primera parte). Nutrición hospitalaria, 21(3), 291-299.

Gonzáles-Torres, L., Téllez-Valencia, A., Sampedro, J. G. y Nájera H. (2007). Las proteínas en la nutrición. RESPYN. Área Académica de Nutrición. Instituto de Ciencias de la Salud, 2(8), 7.

Hernández, T. M. (2004). Recomendaciones nutricionales para el ser humano: actualización. Instituto de Nutrición e Higiene de los Alimentos, 23(4), 66-92.

Herz, W., \& Kulanthaivel, P. (1985). An amide from Salmea scandens. Phytochemestry, 24(1), 173-174.

INCAP (Instituto de Nutrición de Centro América y Panamá). (2007). Tabla de composición de alimentos de Centroamérica. Editorial Guatemala.

INEE (Instituto Nacional para la Evaluación de la Educación).(2014). Consulta previa, libre e informadaparapueblos y comunidades indigenas en materia de evaluación educativa, Buena Vista Loxicha, Pochutla, Oaxaca. México.

Juárez-Castañeda, I. S. (2013). Estudio etnobotánico de plantas y hongos comestibles en San Bartolomé Loxicha y San Vicente Yogondoy, distrito de Pochutla, Oaxaca (tesis de licenciatura). México: Universidad Autónoma Chapingo.

Lara-Cortés, E., Martín-Belloso, O., Osorio-Díaz, P., Barrera-Necha, L. L., Sánchez-López, J. A. y Bautista-Baños, S. (2014). Actividad antioxidante, composición nutrimental y funcional de flores comestibles de Dalia. Revista Chapingo Serie Horticultura, 20(1), 101-116.

Latham, M. C. (2002). Nutrición humana en el mundo en desarrollo. Alimentación y nutrición. Núm. 29. Organización de las Naciones Unidas para la Alimentación y la Agricultura. Roma, Italia.

Lascurain, M., Avendaño, S., Del Amo, S. y Niembro, A. (2010). Guía de frutos silvestres comestibles en Veracruz. Fondo sectorial para la investigación, el desarrollo y la innovación tecnológica forestal. México: CONAFOR-CONACyT.

Luna-José, A. de L. y Rendón-Aguilar, B. (2008). Recursos vegetales útiles en diez comunidades de la Sierra Madre del Sur, Oaxaca, México. Polibotánica, 26, 193-242. 
Martínez, M. (1994). Catálogo de nombres vulgares y cientificos de plantas mexicanas. México: Fondo de Cultura Económica.

Morales, T. M. G. (2007). Uso del dosel de selva mediana subperennifolia con cafetal en Santa Maria Xadani, San Miguel del Puerto, Oaxaca (tesis de maestría). México: Instituto Tecnológico del Valle de Oaxaca.

Murillo, J.y Martínez, C. (2010). Investigación etnográfica, en D. C. Barbolla, M. N. Benavente, B. T. López, G. C. Martín de Almagro, S. L. Perlado y L. C. Serrano, (Eds). Madrid: Universidad Autónoma de Madrid.

Plan Municipal de Desarrollo Rural Sustentable de San Agustín Loxicha, Pochutla, Oaxaca. 2008-2010.

Rendón, A. B., Rebollar, D. S., Caballero, N. J. y Martínez, A. M. A. (2001). Plantas cultura y sociedad. Estudio sobre la relación entre seres humanos y plantas en los albores del siglo XXI. Universidad Autónoma Metropolitana. Secretaria del Medio Ambiente Recursos Naturales y Pesca. México, D. F. p. 315.

Rzedowski, J., Calderón, R. G. y Carrillo-Reyes, P. (2011). Compositae. Tribu Heliantheae II. Fascículo 157. Flora del Bajío y Regiones Adyacentes. México.

Sosa-Montes, M., Martínez-Antonio, F., Cuevas-Reyes, V. y Espejel, G. A. (2007). Contribución de los productos forestales no maderables a la economía familiar en el ejido San José Cieneguilla, Oaxaca. Naturaleza y Desarrollo, 2(2), 21.

Villa-Ruano, N., Zurita-Vásquez, G. G., Pacheco-Hernández, Y., Betancourt-Jiménez, M. G., Cruz-Durán, R., \& Duque-Bautista, H. (2013). Anti-lipase and antioxidant properties of 30 medicinal plants used in Oaxaca, México. Biological Reserch, 46(2), 153-160.

Villa-Ruano, N., Pacheco-Hernández, Y., Rubio-Rosas, E., Lozoya-Gloria, E., Mosso-González, C., Ramón-Canul, L. G., \& Cruz-Durán, R. (2015). Essential oil composition and biological/pharmacological properties of Salmea scandens (L.) DC. Food Control, 57,177-184.

CC BY-NC-ND 